\title{
Influence of Axial and Peripheral Ligands on the Electronic Structure of Titanium Phthalocyanines
}

David F. Pickup, ${ }^{* \dagger, \dagger}$ Ioannis Zegkinoglou, ${ }^{\S}, \|$ Beatriz Ballesteros, ${ }^{\perp}$ Carolina R. Ganivet, ${ }^{\perp}$ J. M. García-Lastra, ${ }^{\dagger, \ddagger} \#$ Peter L. Cook, ${ }^{\nabla}$ Phillip S. Johnson, ${ }^{\S}$ Celia Rogero, ${ }^{\dagger, \bigcirc}$ Frank de Groot,

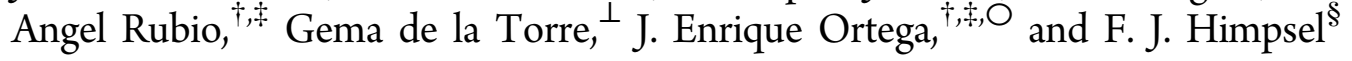

${ }^{\dagger}$ Material Physics Center, Centro de Física de Materiales (CSIC-UPV-EHU), 20018 San Sebastian, Spain

${ }^{\ddagger}$ Departamento de Fisica Aplicada I, Universidad del Pais Vasco, 20018 San Sebastian, Spain

${ }^{\S}$ Department of Physics, University of Wisconsin Madison, 1150 University Avenue, Madison, Wisconsin 53706, United States

"Advanced Light Source, Lawrence Berkeley National Laboratory, Berkeley, California 94720, United States

${ }^{\perp}$ Departamento de Química Orgánica, Facultad de Ciencias, Universidad Autónoma de Madrid, Campus de Cantoblanco, 28049 Madrid, Spain

\#Department of Physics, Center for Atomic-Scale Materials Design, Technical University of Denmark, DK-2800 Kongens Lyngby, Denmark

${ }^{\nabla}$ Natural Sciences Department, University of Wisconsin Superior, Superior, Wisconsin 54880, United States

ODonostia International Physics Center, 20018 San Sebastian, Spain

Inorganic Chemistry and Catalysis, Department of Chemistry, Utrecht University, Universiteitsweg 99, 3584 CG Utrecht, The Netherlands

Supporting Information

ABSTRACT: To discover how molecular changes affect the electronic structure of dye molecules for solar cells, we have investigated four titanium phthalocyanines customized by axial and peripheral ligands (monodentate oxo versus bidentate catechol and tert-butyl versus tert-butylphenoxy, respectively). X-ray absorption spectroscopy and photoelectron spectroscopy were combined with density functional theory (DFT) and crystal-field multiplet calculations to characterize the $\mathrm{Ti} 3 \mathrm{~d}$ and $\mathrm{N} 2 \mathrm{p}$ valence electrons that form the frontier orbitals. When a monodentate oxo axial ligand

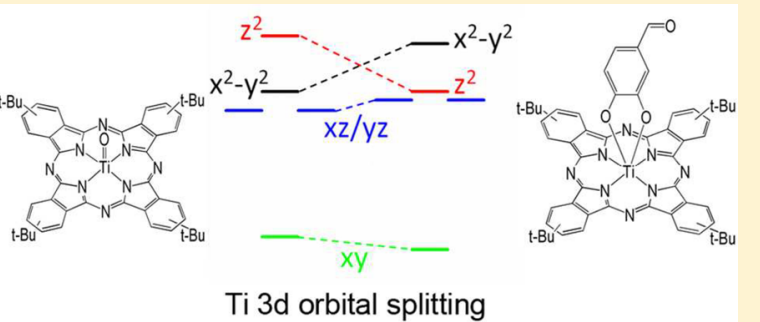
was replaced by a bidentate catechol ligand, the multiplet structure of the Ti 2 p-to-3d transitions was found to change systematically. The most noticeable change was an additional transition into the low-lying $3 \mathrm{~d}_{x y}$ level, which is attributed to a reduction in local symmetry from 4 -fold to 2 -fold at the Ti center. An increase of the Ti $2 p$ core-level binding energy was observed in the bidentate complex and compared to a calculated core-level stabilization. DFT predicts a change of the LUMO from the inner phthalocyanine ring to the $\mathrm{Ti} \mathrm{d}_{x y}$ orbital and a reversal of the high-lying $\mathrm{d}_{x^{2}-y^{2}}$ and $\mathrm{d}_{z}{ }^{2}$ orbitals. The $\mathrm{N}$ 1s edge was calculated using time-dependent density functional theory (TDDFT) and compared to experiment.

\section{INTRODUCTION}

Organic photovoltaics have been the subject of intense research over recent years because of their potential as clean, low-cost alternatives to nonrenewable energy sources. ${ }^{1-4}$ The challenge that the community faces is reducing the cost per watt to become competitive with that of established energy sources. Both the use of purely organic solar cells and the incorporation of organic chromophores into dye-sensitized solar cells (DSSCs) are promising approaches because of the low processing costs of these devices and the possibility of tuning the electronic properties of the organic components to enhance device efficiency. ${ }^{5}$
The light-harvesting and charge-transfer properties observed in complex biomolecules can serve as a guide, and their active centers can be reproduced in simpler molecules such as porphyrins and phthalocyanines. However, accurate determination of the electronic structure of these materials is fundamental to understanding and enhancing their function within the solar cell. To systematically optimize the performance of solar cells, we have embarked on a program to investigate the electronic structure of dye molecules, such as

Received: November 30, 2012

Revised: January 29, 2013

Published: February 5, 2013 


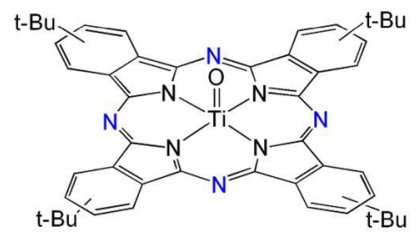

GT1

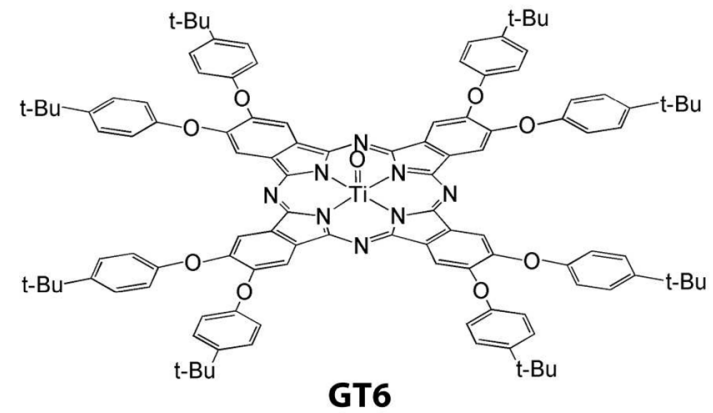

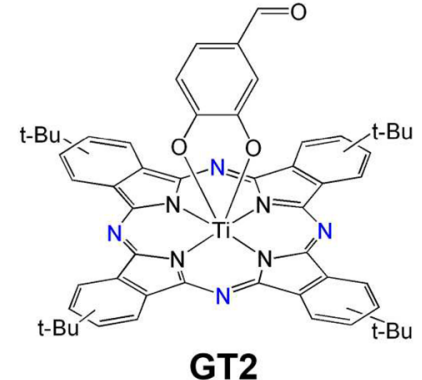

GT2

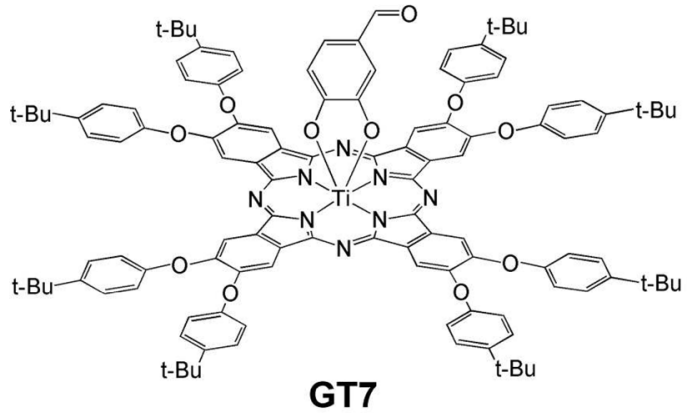

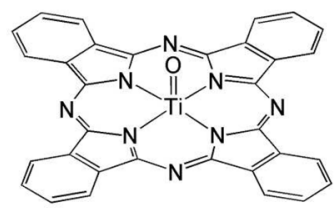

TiOPc

Figure 1. Schematic diagram of the four customized titanium phthalocyanines studied and TiOPc.

phthalocyanines and porphyrins. ${ }^{6-10}$ (For an overview, see ref 11.) In this work, we focus on phthalocyanine $(\mathrm{Pc})$ materials, which have received a great deal of attention because of their stability, high absorption coefficient, and ease of susceptibility to synthetic modification-most notably through the replacement of the central metal ion. ${ }^{12,13}$ At present, the record power conversion efficiency for a phthalocyanine-based DSSC is 5.5\%. ${ }^{14}$ The occupied electronic states of phthalocyanines have been measured by X-ray photoelectron spectroscopy (XPS), ${ }^{15-17}$ whereas their unoccupied states can be probed by inverse photoemission ${ }^{18}$ and near-edge X-ray absorption fine structure (NEXAFS) spectroscopy. ${ }^{6,9,16,19-21}$ By using the absorption edges of the central metal atom and the surrounding $\mathrm{N}$ atoms, one can selectively determine the unoccupied states in different parts of the molecule. The valence-band structure of these molecules can be investigated by employing valence-band photoemission spectroscopy. The levels of the highest occupied molecular orbitals (HOMOs) and lowest unoccupied molecular orbitals (LUMOs) of the organic materials have particular importance in the operation of solar cell devices. They are important in determining the light absorption, whereas charge separation and open-circuit voltage are dependent on the alignment of the energy levels of the acceptor and donor materials.

The incorporation of metal cations into the Pc ring increases the complexity of the electronic structure near the Fermi level $\left(E_{\mathrm{f}}\right)$ because of the overlap of metal $3 \mathrm{~d}$ states with $\mathrm{C}$ and $\mathrm{N} 2 \mathrm{p}$ states from the phthalocyanine ring. The electronic structure of $3 \mathrm{~d}$ metal phthalocyanines has been investigated systematically by X-ray absorption spectroscopy from $\mathrm{Mn}$ through $\mathrm{Zn} .{ }^{6,9,11}$
Whereas manganese and iron phthalocyanines are readily obtained in both the $3+$ and $2+$ oxidation states, the subsequent $3 \mathrm{~d}$ metals occur mainly in the $2+$ oxidation state. Among the early transition metals, $\mathrm{Ti}(\mathrm{IV})$ and $\mathrm{V}(\mathrm{IV})$ phthalocyanines have dominated the literature. ${ }^{20-27}$

In this work, we investigated the electronic properties of Tibased phthalocyanines that were tailored using axial and peripheral ligands. Axial ligands affect mainly the metal atom at the center of the molecule. Peripheral groups can act as linkers of a pthalocyanine dye molecule to an acceptor or donor nanoparticle, but can also modulate the electronic characteristics of the macrocycle. Donor-acceptor substitution in a macrocyclic dye leads to a donor $-\pi$-acceptor complex in which photogenerated charge carriers can be separated efficiently. $^{28-31}$

Specifically, we considered the four molecules depicted in Figure 1. GT1 and GT6 are similar to the well-studied titanyl phthalocyanine TiOPc (also shown for comparison), but they are peripherally functionalized with four tert-butyl and eight electron-donating tert-butylphenoxy groups, respectively. These groups are expected to aid solvation by organic solvents, important in wet processing techniques. GT2 and GT7 are analogous to GT1 and GT6, respectively, apart from the change in axial ligand from an oxo ligand to a catechol-based bidentate ligand, changing the coordination geometry at the central $\mathrm{Ti}$ atom.

XPS and NEXAFS spectroscopy were used to obtain the electronic structures of the molecules and to demonstrate the effects of the ligands, whereas crystal-field multiplet calculations and density functional theory (DFT) were used in tandem to 
interpret the results. We found that incorporating the peripheral electron-donating phenoxy groups in GT6 had little effect on the electronic structure at the Ti $2 \mathrm{p}$ and $\mathrm{N} 1$ s edges with regard to the tert-butyl-substituted GT1 compound. Changing the axial ligand from the monodentate oxo ligand in GT1 to the bidentate catechol ligand in GT2 (and similarly for GT6 and GT7) reduced the local symmetry at the Ti ion from 4-fold to 2-fold, which was found to allow an additional transition into the low-lying $3 \mathrm{~d}_{x y}$ orbital. An increase in the binding energy of the Ti $2 p_{3 / 2}$ core level of the bidentate ligand was observed by XPS. DFT predicted that the exchange of the axial ligand shifted the location of the LUMO and changed the order of the $\mathrm{d}_{x^{2}-y^{2}}$ and $\mathrm{d}_{z}{ }^{2}$ orbitals between GT1 and GT2.

\section{EXPERIMENTAL SECTION}

Synthesis of GT1 and GT2 has already been reported elsewhere. ${ }^{32-34}$ Details on the synthesis of GT6 and GT7 dyes are described in the Supporting Information.

Sample Preparation. Three different techniques were employed to prepare samples for NEXAFS measurements: (1) embedding the sample powder in carbon tape, (2) evaporating a thin film onto Au-coated $\mathrm{Si}(111)$ wafers under ultrahighvacuum (UHV) conditions, (3) drop-casting from solutions in different organic solvents [dichloromethane (DCM), tetrahydrofuran (THF)] onto Au-coated wafers. We observed that the NEXAFS results were sensitive to the preparation conditions, particularly the Ti $2 \mathrm{p}$ edge spectra. Adhering to the following preparation guidelines for drop-cast samples yielded spectra consistent with the powder samples:

(1) Prepare fresh sample solutions and ensure that they are homogeneous

(2) Use anhydrous (or extremely low- $\mathrm{H}_{2} \mathrm{O}$-content) solvents.

(3) Use low dye concentrations $(1 \mathrm{mg} / \mathrm{mL})$

(4) Drop-cast the solution onto the substrate just before introducing it into the vacuum chamber.

Figure 2 compares Ti $2 p$ spectra of GT1 in powder and drop-cast form for which these procedural guidelines were

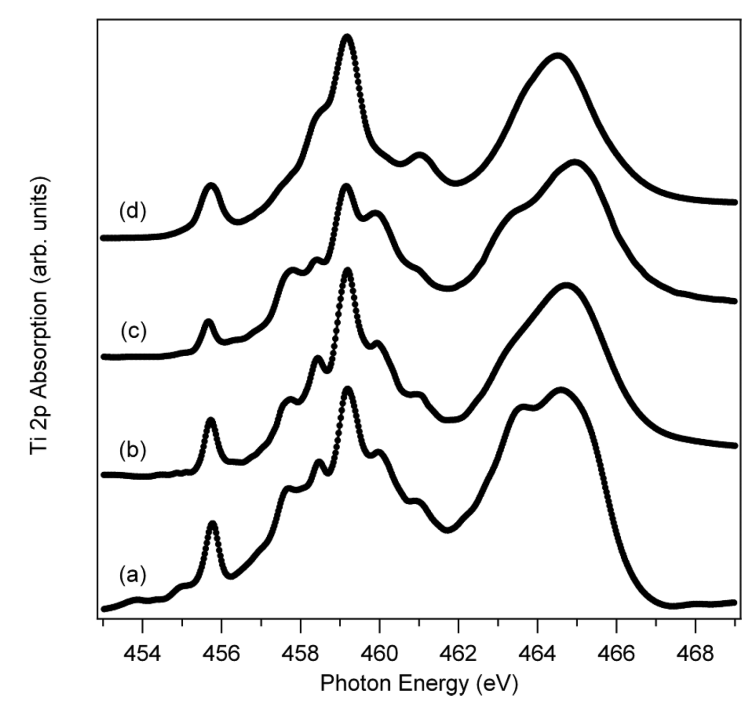

Figure 2. Comparison of the Ti $2 p$ edge spectra of a GT1 (a) powder sample, (b) drop-cast sample from DCM solution, (c) drop-cast sample from THF solution, and (d) evaporated sample prepared under UHV conditions. followed, as well as data from an evaporated sample. All of the spectra exhibited peak positions at almost identical photon energies. However, the relative intensities of the peaks varied depending on the deposition method, with the strongest similarity observed between the powder and DCM-drop-cast samples. In all cases, the peak at $459 \mathrm{eV}$ was the most intense. The intensity of the low-energy peak in the THF-drop-cast sample decreased, whereas the peaks at 457.7 and $459.8 \mathrm{eV}$ were more pronounced. This is in contrast to the UHVevaporated sample, which displayed relatively low-intensity features at 457.7 and $459.8 \mathrm{eV}$. The increased intensity of these peaks in the THF-drop-cast sample might be the result of a small amount of water absorbed during sample preparation because of the hygroscopic nature of the solvent. The lower intensity of these peaks in the evaporated sample indicate a film containing less water, as would be expected.

Deviation from the mentioned guidelines by using old solutions, nonanhydrous solvents, or solutions of higher concentrations resulted in the loss of fine structure, giving broader, featureless Ti $2 p$ spectra, as highlighted in Figure 3.

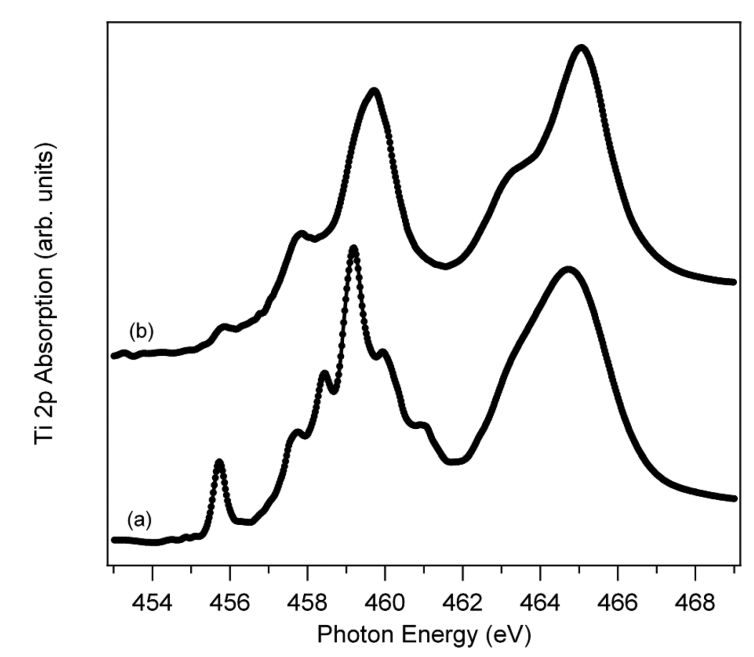

Figure 3. Comparison of the GT1 Ti $2 p$ edge spectra of (a) a wellprepared drop-cast sample and (b) a drop-cast sample prepared using a 2-day-old nonanhydrous THF solution.

Possible explanations for these spectral changes include water/ solvent coordination at the $\mathrm{Ti}$ center, ligand exchange/loss, and/or aggregation of the molecules. The spectrum of the poorly prepared sample in Figure $3 \mathrm{~b}$ has a multiplet structure similar to that of $\mathrm{Ti}^{4+}$ in an octahedral environment, with its typical $e_{g}-t_{2 g}$ splitting of the two spin-orbit components. Such spectra are found for titanates in which $\mathrm{Ti}^{4+}$ is surrounded by an octahedral cage of oxygen atoms. ${ }^{35,36}$ However, the large inhomogeneous broadening in Figure $3 \mathrm{~b}$ indicates that solvent reactions did not lead to a unique structure. For a full understanding of the effects of sample preparation, further investigation is required, which is beyond the scope of this work.

We also produced thin films of GT1 and GT2 by sublimation in ultrahigh vacuum from a tantalum Knudsen cell onto Aucoated $\mathrm{Si}(111)$ wafers. The typical sublimation temperature was about $430{ }^{\circ} \mathrm{C}$. GT6 and GT7 decomposed before significant amounts evaporated. That is, the $\mathrm{N} 1 \mathrm{~s}$ spectra showed a peak at $399.9 \mathrm{eV}$ corresponding to nitrile fragments. ${ }^{37}$

Measurements. NEXAFS data provide details about the electronic structure of materials by exciting electrons from the 
core levels to unoccupied valence-band orbitals, such as the LUMO, which is populated when an organic dye absorbs photons of certain wavelengths. NEXAFS spectroscopy at the $2 p$ absorption edge of titanium and the 1 s absorption edge of nitrogen was employed to characterize the phthalocyanine dyes. The measurements were conducted at two different synchrotron light sources, using Beamline 8.0.1 of the Advanced Light Source (ALS) at the Lawrence Berkeley National Laboratory and the VLS PGM Beamline at the Synchrotron Radiation Center (SRC) in Madison, WI. Radiation damage was minimized by working with the narrowest possible monochromator slits, using filters (at the SRC), or spreading the light out over an area of $3 \times 5 \mathrm{~mm}^{2}$ (at the ALS). All absorption spectra were measured by collecting the total electron yield. The energy resolution was better than $0.2 \mathrm{eV}$ at all edges. The photon energy was calibrated at the Ti $2 \mathrm{p}$ and $\mathrm{N}$ 1s edges using the sharp $2 \mathrm{p}$-to- $3 \mathrm{~d}$ transition in rutile $\mathrm{TiO}_{2}$ powder at 458.0 $\mathrm{eV},{ }^{35}$ with the $\mathrm{N}$ 1s-to- $\pi^{*}$ transition of $\mathrm{N}_{2}$ as the primary standard. ${ }^{38}$

Complementary XPS data were collected at the Materials Physics Center in San Sebastian, Spain. Samples were prepared by embedding the dye powders in carbon tape. The data were obtained using a Phoibos photoelectron spectrometer equipped with an $\mathrm{Al} \mathrm{K} \alpha \mathrm{X}$-ray source (1486.6 eV photon energy) as the incident photon radiation. The overall resolution was approximately $0.9 \mathrm{eV}$. The pressure in the analyzing chamber was $<2.0 \times 10^{-9}$ mbar during measurements. Spectra were referenced to the adventitious $\mathrm{C} 1 \mathrm{~s}$ signal at $284.8 \mathrm{eV}$.

Calculations. Interpretation of the multiedge NEXAFS results from the less complex GT1 and GT2 molecules was carried out using a combination of DFT and CTM4XAS crystal-field atomic multiplet calculations. The fine structure of the transition-metal $2 \mathrm{p}$ edge is strongly influenced by atomic multiplet splitting between the $3 \mathrm{~d}$ valence electrons and the $2 \mathrm{p}$ core hole. Unlike other phenomena, these atomic multiplet effects are not screened in the solid state and must be considered to obtain an accurate description of the NEXAFS $2 p$ edge. DFT is also known to overestimate the covalence between transition-metal atoms and ligands, which can lead to errors in the intensities of the corresponding transitions. For these reasons, we decided to simulate the fine structure of the Ti $2 p$ edge using the CTM4XAS program, which was designed for atomic multiplets. DFT calculations were used to obtain starting values of the crystal-field parameters for the CTM4XAS fit to the data. These parameters determine the sequence of the Ti 3d valence states. DFT also provides the wave functions and energies of unoccupied orbitals. The $\mathrm{N}$ 1s edge is less affected by interactions with the core hole than the $\mathrm{Ti} 2 \mathrm{p}$ edge. Therefore, we used time-dependent DFT (TDDFT) calculations for a comparison with the experimental data at the $\mathrm{N} 1 \mathrm{~s}$ edge.

DFT calculations were carried out by means of the Amsterdam density functional (ADF) code. ${ }^{39}$ All atoms were described through basis sets of TZP quality (triple- $\zeta$ STO plus one polarization function) given in the program database, including all of the core electrons in the calculation (i.e., with no frozen-core approximation). The exchange-correlation energy was computed according to the generalized gradient approximation (GGA) by means of the Perdew-BurkeErnzerhof (PBE) functional. ${ }^{40}$ The results were checked using the local density approximation (LDA) by means of the Vosko-Wilk-Nussair (VWN) functional ${ }^{41}$ and a hybrid functional (BHandHLYP). ${ }^{39}$ The first step of the calculations consisted of obtaining the structures of the different phthalocyanines in their ground state. The structures were relaxed until a maximum force below $0.04 \mathrm{eV} / \AA ̊$ was obtained. In a second step, the NEXAFS transitions were calculated using the TDDFT method. As was expected, there was a shift between the TDDFT-calculated and experimental spectra. Although the smallest shift was obtained with the BHandHLYP hybrid functional, the relative positions of the different peaks in the spectra were better reproduced with the PBE functional. For this reason, we used the PBE results for the peak assignment and analysis of the $\mathrm{N} 1$ s edge experimental spectra.

The Ti $2 p$ edge spectra of GT1 and GT2 were simulated using the semiempirical CTM4XAS program of de Groot and Stavitski ${ }^{42}$ designed to simulate the $2 \mathrm{p}$ edges of transition-metal complexes. The $2 \mathrm{p}$ X-ray absorption edge of transition-metal complexes are strongly affected by the significant overlap of the $2 p$ core and $3 d$ valence wave functions. The CTM4XAS program is based on Cowan's atomic multiplet code ${ }^{43}$ with subsequent modifications by Butler, ${ }^{44}$ Thole et al., ${ }^{45}$ and Ogasawara et al. ${ }^{46}$ incorporating crystal-field and chargetransfer effects. The CTM4XAS program requires the input of several parameters to calculate the absorption edge. After selection of $\mathrm{Ti}$ in its 4+ oxidation state, the symmetry of the complex is required. However, at the moment, the CTM4XAS program does not have the option of directly calculating lowsymmetry complexes such as those studied in this work. As such, we selected the $D_{4 h}$ (square-planar) symmetry system, typical of unsubstituted phthalocyanines, and input the crystalfield parameters $(D q, D t$, and $D s)$ to approach the system we were simulating. These parameters were initially obtained from DFT calculations and subsequently optimized to obtain better agreement with experimental data.

\section{RESULTS AND DISCUSSION}

Background. GT1 has a chemical structure very similar to that of the well-studied TiOPc, except for the weakly electrondonating tert-butyl groups at the periphery of the phthalocyanine ring. The central $\mathrm{Ti}$ ion exhibits the same coordination geometry in both GT1 and TiOPc. In both cases, the Ti ion exhibits a 5-fold distorted square-pyramidal coordination geometry (distorted $C_{4 v}$ point group), with the $\mathrm{Ti}$ atom situated slightly out of the plane of the phthalocyanine ring, typically exhibiting an $\mathrm{O}-\mathrm{Ti}-\mathrm{N}$ angle of $110^{\circ} .47$ The inner nitrogen-containing ring of the phthalocyanine ligand will largely determine the frontier orbitals ${ }^{48}$ and, thereby, the optical absorption of the dye molecule.

Because of the analogous structures of the two materials, GT1 was found to exhibit electronic properties similar to those of TiOPc. The XPS data were comparable, with a $0.2 \mathrm{eV}$ global shift to lower binding energy for GT1, as highlighted in Table 1. The X-ray absorption results from the $\mathrm{Ti} 2 \mathrm{p}$ edge and $\mathrm{N} 1 \mathrm{~s}$ edge were also similar to previously published TiOPc data ${ }^{20,21}$ and are discussed in more detail in subsequent sections. Previous research showed that the HOMO of TiOPc is mainly associated with the phthalocyanine ligand. ${ }^{48,49}$ DFT calculations for GT1 (using $C_{4 v}$ symmetry) agree with this assignment, as seen in the corresponding molecular orbital wave functions in Figure 4, where the HOMO is mainly located on the inner pyrrolic carbons. The same result was found for GT2 using $C_{s}$ symmetry, also depicted in Figure 4 . It is worth pointing out that the aldehyde group attached to the catechol phenyl ring in GT2 (and GT7) introduces asymmetry that reduces the global symmetry of the molecules to $C_{s}$ symmetry. 
Table 1. XPS Core-Level Binding Energies (eV) for the Four Studied Complexes, along with Literature TiOPc Values

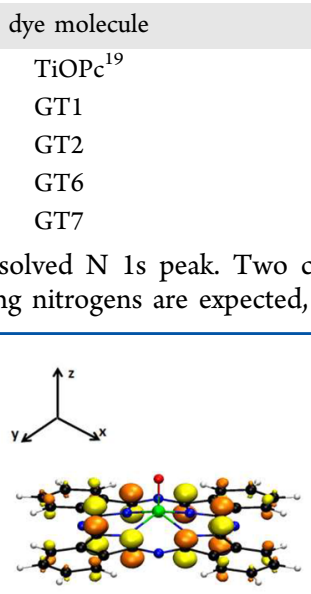

13 a2

HOMO GT1

$\mathrm{N} 1 \mathrm{~s}^{a}$
399.2
399.0
399.0
398.9
391.1

391.1

Ti $2 \mathrm{p}_{3 / 2}$
458.4
458.1
458.6
458.2
458.7

${ }^{a}$ Unresolved $\mathrm{N}$ 1s peak. Two components due to pyrrolic and azabridging nitrogens are expected, plus shakeup satellites.

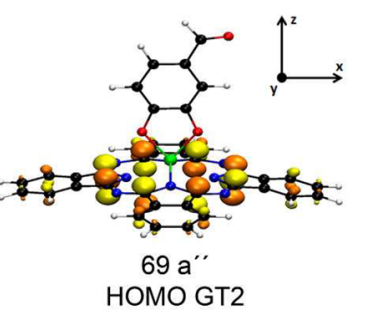

Figure 4. Wave functions of the highest occupied molecular orbitals (HOMOs) of GT1 ( $C_{4 v}$ symmetry) and GT2 ( $C_{s}$ symmetry). The orbitals are labeled according to symmetry assignment.

If one considers only the nearest neighbors of the central $\mathrm{Ti}$ atom, the local symmetry is $C_{2 v}$. For GT1 and GT2, the HOMO has the same character as one of the Gouterman orbitals predicted for square-planar phthalocyanines. ${ }^{50-52}$

The following section focuses on how changing the axial ligand and introducing electron-donating peripheral groups affect the electronic structures of different parts of the dye molecules. We separate the discussion into two sections: First, we consider the effects at the central titanium cation using $\mathrm{Ti}$ $2 p$ edge data, and subsequently, we analyze the surrounding phthalocyanine ring using $\mathrm{N}$ 1s edge data.

NEXAFS Titanium 2p Edge. The spectral features observed in the Ti $2 p$ edge spectra correspond to transitions from the spin-orbit-split Ti $2 \mathrm{p}$ states to the conduction band of the molecule. The spectral features can be separated into two groups: The group of peaks from 456 to $461 \mathrm{eV}$ is assigned to the $2 \mathrm{p}_{3 / 2}$ part of the manifold, whereas the group between 461 and $468 \mathrm{eV}$ is due to the $2 \mathrm{p}_{1 / 2}$ part. The coordination geometry around the central metal ion is one parameter that is known to strongly affect the X-ray absorption $2 \mathrm{p}$ edge of transition-metal complexes. ${ }^{53}$ This is highlighted in Figure 5, which shows the Ti $2 \mathrm{p}$ absorption edges of rutile $\mathrm{TiO}_{2}, \mathrm{GT} 1$, and GT2. In all cases, the $\mathrm{Ti}$ ion is in the 4+ oxidation state, but the different coordination symmetries around the Ti ion result in significant differences in the fine structure at the $2 p$ edge. It is worth noting that Krüger showed that the rutile $\mathrm{TiO}_{2}$ fine structure is also strongly influenced by a long-range band-structure effect (over a 1-nm length scale) that causes the splitting of the feature centered at $460.2 \mathrm{eV}{ }^{54}$ This type of long-range effect has not been found to have a significant influence on the fine structure of $\mathrm{Ti}$ complexes such as TiOPc, where the crystal field due to the nearest neighbors of the central $\mathrm{Ti}$ atom is most important. $^{55}$

The upper panel of Figure 6 shows the Ti 2p edge NEXAFS spectra of the four customized complexes studied in this work. The GT1 spectrum exhibits well-resolved peak structure in the $2 \mathrm{p}_{3 / 2}$ manifold with features similar to those in previously published data for TiOPc. ${ }^{20,21}$ Comparison of the spectra from

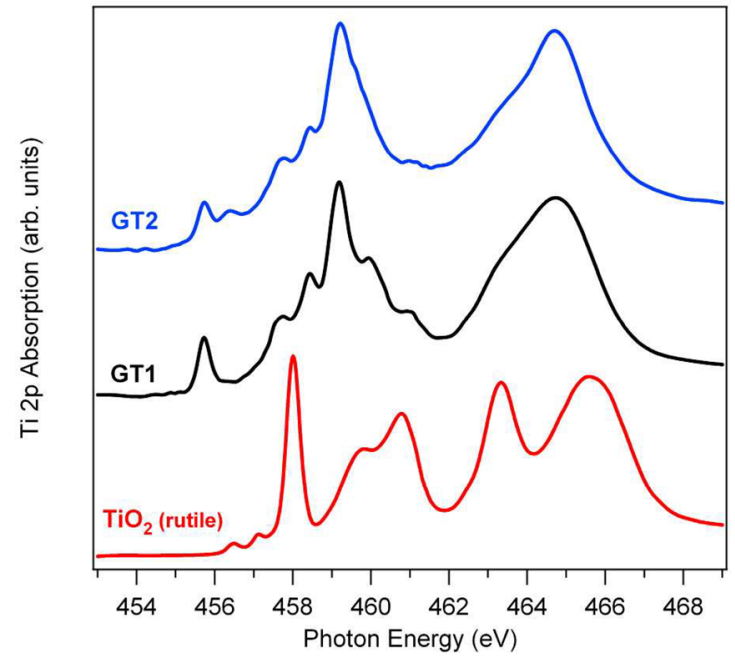

Figure 5. Comparison of $\mathrm{Ti} 2 \mathrm{p}$ edge of rutile $\mathrm{TiO}_{2}, \mathrm{GT} 1$ and GT2. In all complexes titanium is in the 4+ oxidation state, but differences in the nature of the ligands and coordination geometries result in distinct Ti $2 p$ edges.

the four molecules shows that the pair GT1/GT6 exhibits very similar features and peak positions, and likewise for GT2/GT7. These pairs of molecules contain the same axial ligands, indicating that the peripheral groups have less influence at the Ti $2 p$ edge, as one might expect from their large distance from the central $\mathrm{Ti}$ atom.

Whereas the pairs of molecules with identical axial ligands showed very similar $\mathrm{Ti} 2 \mathrm{p}$ spectra, significant differences appeared when the axial ligand was changed. The most visible difference is an extra peak appearing for GT2/GT7 about 0.6 $\mathrm{eV}$ above the lowest transition. The latter can be assigned to a transition into the $\mathrm{Ti} 3 \mathrm{~d}_{x y}$ orbital (see Figure 7 ). The additional peak for GT2/GT7 is likely to have $3 d_{x y}$ symmetry as well, because there are no other $3 \mathrm{~d}$ levels nearby. A possible explanation for the extra peak is the reduced symmetry at the central Ti atom of GT2/GT7, which is 2-fold instead of the 4fold symmetry of GT1/GT6. Transitions that are symmetryforbidden in 4-fold symmetry can become allowed in the lower 2 -fold symmetry. Such extra transitions in reduced symmetry have been found previously at the $\mathrm{Ca} 2 \mathrm{p}$-to- $3 \mathrm{~d}$ edge of the $\mathrm{CaF}_{2}(111)$ surface compared to the bulk, using a predecessor of the CTM4XAS program. ${ }^{56}$ Indeed, using the lower C4 symmetry option available in the CTM4XAS program, we did find an extra peak at the location of the additional peak for GT2/GT7.

The lower panel of Figure 6 shows the $\mathrm{Ti} 2 \mathrm{p}$ edge experimental spectra of GT1 and GT2 alongside the CTM4XAS simulated spectra. The agreement between the experimental and calculated data is quite good in both cases: The overall structure and peak positions are reproduced well, whereas the relative peak intensity is not always consistent. Crystal-field parameters $(D q, D s, D t)$ were introduced to simulate the experimental spectra. Initial values for these parameters were obtained from DFT calculations and then subsequently modified to improve the simulation. The values used in the CTM4XAS program for the two simulations are detailed in Table 2.

Removing the multiplet effects in the CTM4XAS program, by setting the Fpd, Gpd, and valence spin-orbit coupling parameters to zero, allows a good approximation of the $\mathrm{d}$ - 

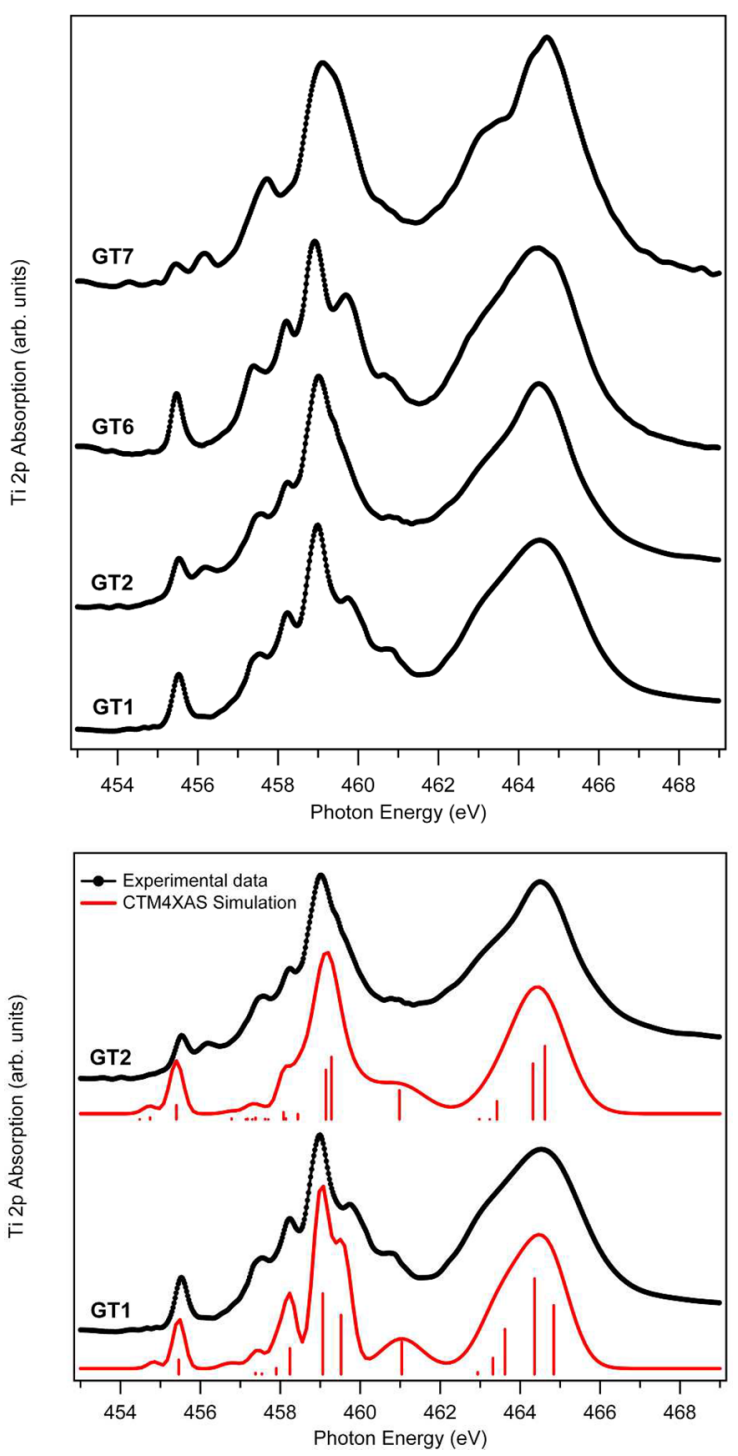

Figure 6. (Top) Comparison of experimental NEXAFS Ti $2 p$ edge spectra of the four studied dye molecules. (Bottom) GT1 and GT2 experimental spectra alongside calculated CTM4XAS spectra.

orbital splitting experienced by each molecule to be uncovered. ${ }^{57}$ It should be noted that setting these parameters to zero removes not only multiplet effects but also any multielectron transitions due to symmetry mixing. Figure 7 shows plots generated by the CTM4XAS program with these effects turned off, where the stick plots correspond to the dorbital splitting as calculated using eqs $1-4 .^{58-60}$ The upper part of the figure allows better direct comparison of GT1 and GT2 splitting.

$$
\begin{aligned}
& \mathrm{b}_{1 \mathrm{~g}}\left(\mathrm{~d}_{x^{2}-y^{2}}\right)=+6 D q+2 D s-1 D t \\
& \mathrm{a}_{1 \mathrm{~g}}\left(\mathrm{~d}_{z^{2}}\right)=+6 D q-2 D s-6 D t \\
& \mathrm{e}_{\mathrm{g}}\left(\mathrm{d}_{x z, y z}\right)=-4 D q-1 D s+4 D t \\
& \mathrm{~b}_{2 \mathrm{~g}}\left(\mathrm{~d}_{x y}\right)=-4 D q+2 D s-1 D t
\end{aligned}
$$

For both GT1 and GT2, the small leading peak at $455.7 \mathrm{eV}$ is assigned to a transition to an orbital with strong $\mathrm{d}_{x y}$ character; this will also be the case for GT6 and GT7. This is clearly seen
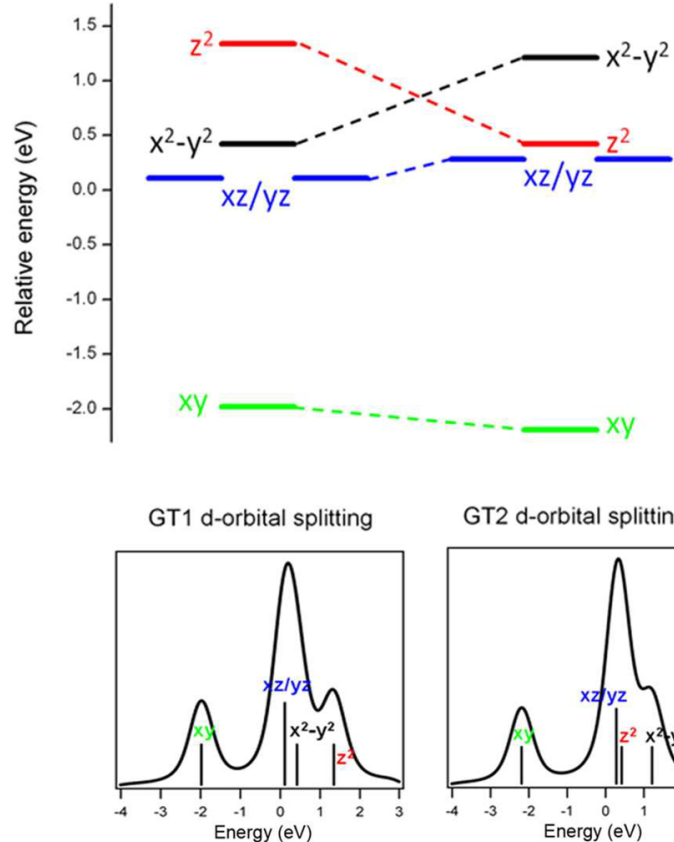

GT2 d-orbital splitting

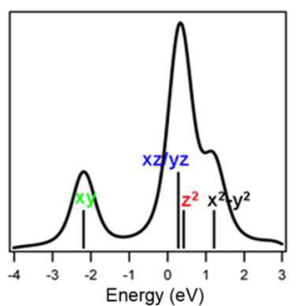

Figure 7. Ti d-orbital splitting for GT1 and GT2 complexes calculated using eqs 1-4 and parameters used in the CTM4XAS simulation of the Ti $2 p$ edge. Bottom: Calculated CTM4XAS spectra for GT1 and GT2 with multiplet effects turned off. The four states generated as a result of the d-orbital splitting are assigned.

Table 2. Input Values Used for CTM4XAS Simulation of GT1 and GT2 Ti 2p Edge Absorption Spectra

\begin{tabular}{lcl} 
& GT1 & GT2 \\
Fdd & Slater Integral Reduction & \\
Fpd & 1.0 & 1.0 \\
Gpd & 0.77 & 0.85 \\
& 0.73 & 0.7 \\
$10 D q$ & Crystal-Field Parameters (eV) & \\
$D t$ & 2.4 & 3.4 \\
$D s$ & 0.16 & 0.35 \\
& -0.43 & -0.24 \\
\hline
\end{tabular}

in Figure 7, where the $d_{x y}$ orbitals of both GT1 and GT2 are significantly stabilized with respect to the other $\mathrm{d}$ orbitals.

TDDFT assigns the $\mathrm{d}_{x y}$ orbital in GT1 to the LUMO + 1 with the LUMO situated on the inner phthalocyanine ring, as shown by the orbital wave functions in Figure 8. DFT predicts that the change in axial ligand from GT1 to GT2 results in a shift in the location of the LUMO, from the inner phthalocyanine ring to the $\mathrm{Ti} \mathrm{d}_{x y}$ orbital. The wave function of the LUMO of GT2 is shown in Figure 9. DFT rationalizes the change in location of the LUMO by a Ti $2 p$ core-level stabilization of $1.2 \mathrm{eV}$ from GT1 to GT2. A similar stabilization was observed experimentally by XPS, although to a smaller extent, with a $0.5 \mathrm{eV}$ shift in the $\mathrm{Ti} 2 \mathrm{p}_{3 / 2}$ core-level peak to higher binding energy from GT1 to GT2 (see Table 1) measured with respect to the Fermi level. However, in the XPS measurements, the energies were referenced to the Fermi level, whereas the calculation was referenced to the vacuum level. A measurement of the vacuum level or a calculation of the Fermi level would be required to directly compare experiment with theory. The position of the Fermi level in the HOMO-LUMO gap depends sensitively on extrinsic gap states, which pin the Fermi level. ${ }^{7}$ The stabilization of the core level is rationalized in 

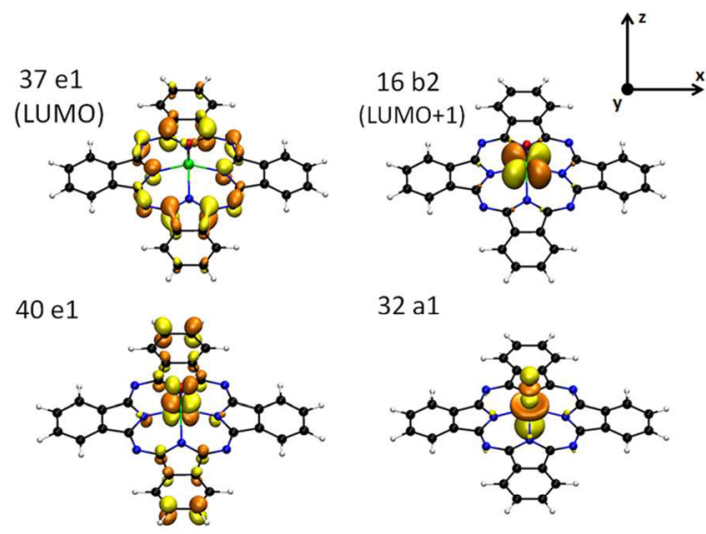

32 a1
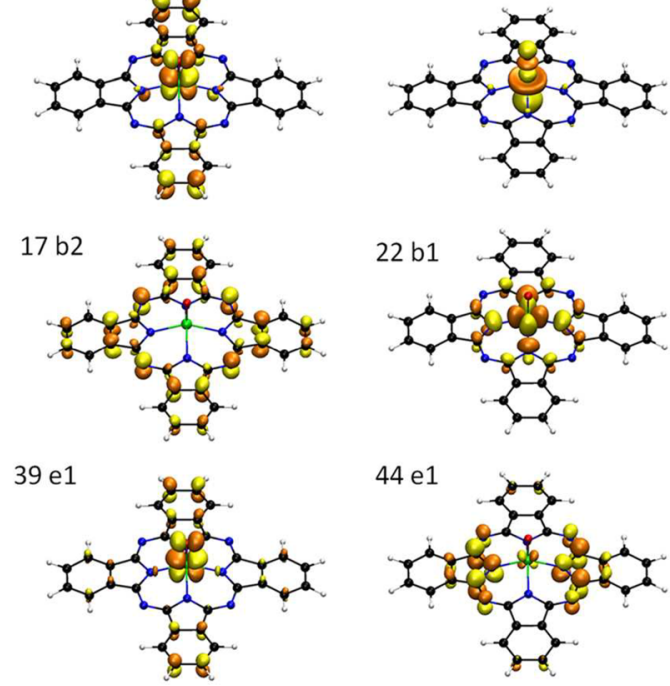

Figure 8. Wave functions of unoccupied molecular orbitals involved in important GT1 transitions. GT1 is assigned $C_{4 v}$ symmetry, and the orbitals are labeled accordingly.

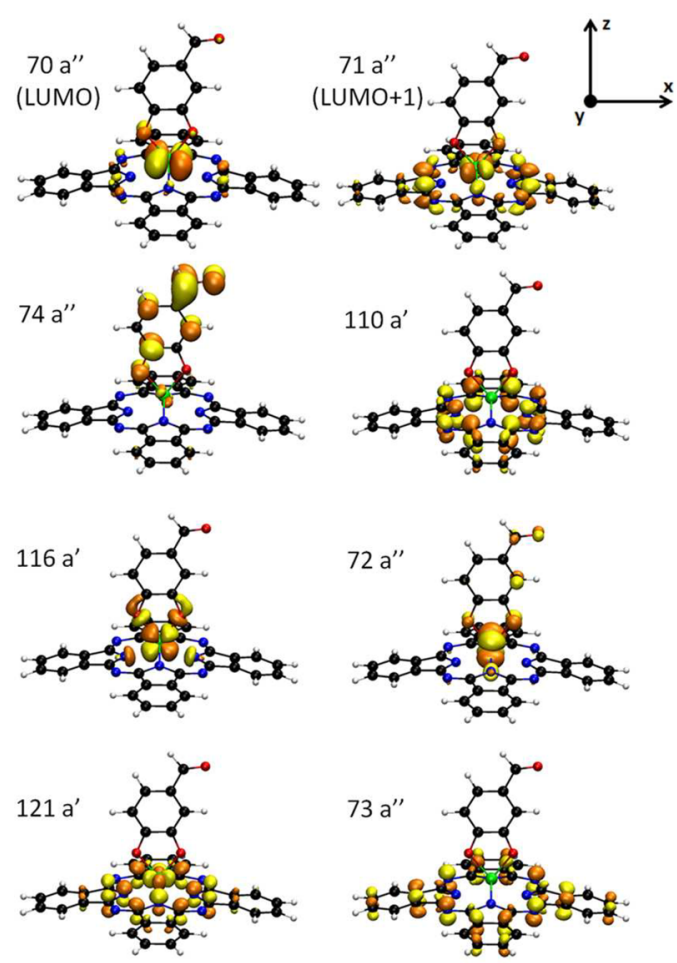

Figure 9. Wave functions of unoccupied molecular orbitals involved in important GT2 transitions. GT2 is assigned $C_{s}$ symmetry, and the orbitals are labeled accordingly.

the DFT calculation by increased positive charge on the GT2 $\mathrm{Ti}$ ion. This can be related to the increased displacement of the
Ti ion out of the plane of the phthalocyanine ring. In GT1, the Ti ion is situated $0.65 \AA$ above the plane, whereas the bulky nature of the axial ligand in GT2 raises the Ti ion to $0.95 \AA$ above the plane of the Pc ring. This will result in less orbital overlap with the electron-rich phthalocyanine ring. Table 1 shows a similar Ti $2 \mathrm{p}_{3 / 2}$ core-level shift from GT6 to GT7. One can also see a decrease in the intensity of the low-energy $\mathrm{d}_{x y}$ peak for GT2 and even more so for GT7 with respect to GT1 and GT6. This can be attributed to the decreased interaction between the $\mathrm{d}_{x y}$ orbital and the nitrogen ring or to a transfer of spectral weight to the extra peak on the high-energy side.

The subsequent peaks found at around 457.7 and $458.4 \mathrm{eV}$ are expected to be due to transitions involving mainly the $d_{x z}$ $\mathrm{d}_{y z}$ orbitals. The lower symmetry of GT1 and GT2 can cause a splitting of the $d_{x z}$ and $d_{y z}$ orbitals, which are degenerate in the $D_{4 h}$ symmetry of the multiplet calculation.

Interestingly, both DFT and subsequent CTM4XAS calculations indicate that there is a reversal in the order of the high-lying $\mathrm{d}_{x^{2}-y^{2}}$ and $\mathrm{d}_{z}{ }^{2}$ orbitals in GT1 and GT2 as seen in Figure 7. This can be explained by taking into account the different coordination of the axial ligand. GT1 has the doublebonded oxo ligand bound directly along the $z$ axis. The short bond results in strong interactions, thereby destabilizing the $d_{z}{ }^{2}$ orbital, whereas the $\mathrm{d}_{x^{2}-y^{2}}$ orbital is slightly stabilized relative to that in standard $D_{4 h}$ complexes because of the displacement of the $\mathrm{Ti}$ ion out of the plane of the ring. Replacement of the axial oxo ligand by the bidentate catechol-based ligand of GT2 results in the loss of strong antibonding interactions directly along the $z$ axis, causing a significant stabilization of the $\mathrm{d}_{z^{2}}$ orbital. The antibonding interactions of the $\mathrm{d}_{x^{2}-y^{2}}$ orbital with nitrogen orbitals on inner phthalocyanine ring is slightly reduced in GT2 as the Ti ion is located farther out of the plane of the ring; however, there is a destabilizing effect because of the interaction with the axial ligand, resulting in an overall increase in energy relative to GT1.

NEXAFS Nitrogen 1s Edge. The electronic structure of the phthalocyanine ring was investigated using the $\mathrm{N} 1 \mathrm{~s}$ absorption edge. TDDFT was used to calculate GT1 and GT2 experimental data. The upper panel of Figure 10 compares the experimental $\mathrm{N}$ 1s NEXAFS data from the four dye molecules. The spectra from the different molecules are very similar and consistent with published data for TiOPc. ${ }^{20,21}$ The position of the low-energy peak, at $398.1 \mathrm{eV}$, is unchanged in all spectra. The only noticeable difference is the intensity ratio of the double feature centered at $399.9 \mathrm{eV}$. This feature was found to be radiation-sensitive, with the ratio changing from scan to scan, as highlighted in Figure S1 (Supporting Information).

The lower panel of Figure 10 shows a plot of the $\pi^{*}$ region of the experimental $\mathrm{N}$ 1s edge spectra of GT1 and GT2 alongside complementary TDDFT-generated data. Tables 3 and 4 summarize the calculated resonance energies, their assignments to specific transitions, and the main states involved in the transitions for GT1 and GT2, respectively. The origin of the transitions is assigned to one of the two distinct $\mathrm{N}$ environments present in the molecules: $\mathrm{N} 1$ signifies the four nitrogens bound to the central $\mathrm{Ti}$ ion (black in Figure 1), whereas N2 indicates the bridging nitrogens (blue in Figure 1). Figures 8 and 9 show wave functions of the unoccupied orbitals involved in the transitions for GT1 and GT2, respectively. The experimental and calculated spectra of GT1 are in reasonable agreement. Calculations show that the intense low-energy peak arises as a result of two transitions, both into the LUMO $\left(37 \mathrm{e}_{1}\right.$ orbital, Figure 8 ) of the molecule, which is a $\pi$ orbital entirely 

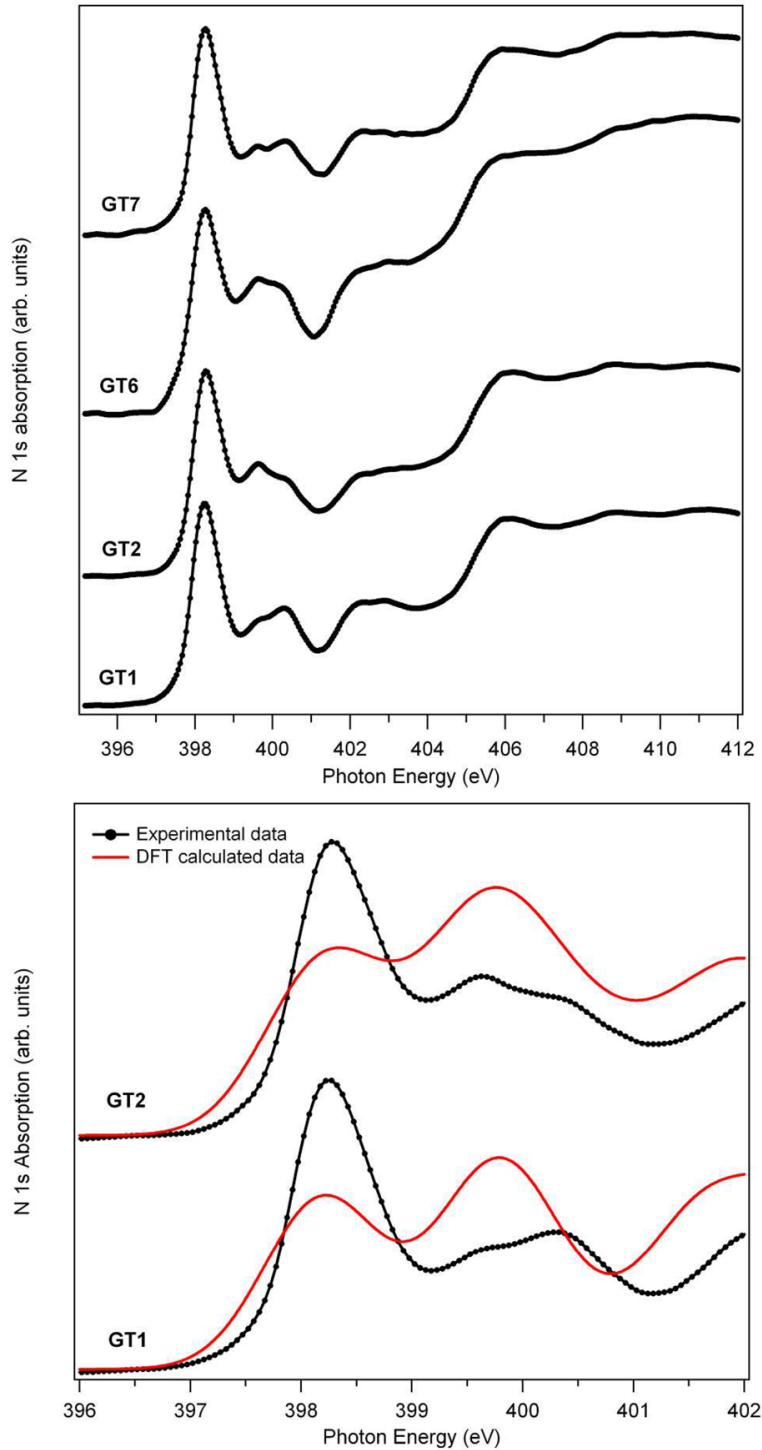

Figure 10. (Top) Comparison of experimental N 1s spectra of the four studied dye molecules. (Bottom) $\pi^{*}$ region of the GT1 and GT2 experimental spectra alongside TDDFT calculations.

located on the inner pyrrole rings (including the $\mathrm{N}$ atoms) of the phthalocyanine structure. There are two transitions each from a different core level situated on the different types of nitrogen ( $\mathrm{N} 1$ and N2). Our assignment of the LUMO to the inner core of the Pc ring is in good agreement with previously published data for TiOPc. ${ }^{21,44}$ The lower-energy resonance of the double feature centered at $399.9 \mathrm{eV}$ is assigned to two excitations, again one from each $\mathrm{N}$ environment: $\mathrm{N} 2$ into a $\pi$ orbital again located on the inner core of the Pc ring $\left(17 b_{2}\right)$ and $\mathrm{N} 1$ to an orbital $\left(19 \mathrm{~b}_{1}\right)$ with character from the inner part of the $\mathrm{Pc}$ ring and from $\mathrm{Ti} \mathrm{d}_{x^{2}-y^{2}}$. The higher-energy part of the feature is due to a transition from N1 into a $\pi$ orbital with character very similar to that of the LUMO.

The lower panel of Figure 10 also demonstrates that the experimental and calculated spectra for GT2 agree well. The first peak is mainly be due to a transition into orbital $110 a^{\prime}$ of the molecule, again a $\pi^{*}$-orbital located on the inner part of the Pc ring. However, there are also contributions from transitions to orbital 71a" (Figure 9), which, although mainly located on the pyrrole rings, does have slight $\mathrm{Ti} \mathrm{d}_{x y}$ character. Our
Table 3. DFT-Calculated Resonance Energies and Relative Intensities for the N 1s Edge of GT1 ( $C_{4 v}$ Symmetry), with Assignments to Major Orbital Transitions and to Different Parts of the Molecule

\begin{tabular}{|c|c|c|c|}
\hline $\begin{array}{l}\text { energy } \\
(\mathrm{eV})\end{array}$ & $\begin{array}{l}\text { relative } \\
\text { intensity }\end{array}$ & orbital transition $^{a}$ & interpretation \\
\hline 398.0 & 0.8 & $\begin{array}{l}3 \mathrm{e} \rightarrow 37 \mathrm{e}(100 \%) \\
\mathrm{N} 2\end{array}$ & P- $\pi$ with $\mathrm{N}$ \\
\hline 398.3 & 0.5 & $\begin{array}{l}2 \mathrm{e} \rightarrow 37 \mathrm{e}(100 \%) \\
\mathrm{N} 1\end{array}$ & $\mathrm{P}-\pi$ with $\mathrm{N}$ \\
\hline 399.4 & 0.6 & $\underset{\mathrm{N} 2}{1 \mathrm{~b}_{2}} \rightarrow 17 \mathrm{~b}_{2}(98 \%)$ & $\mathrm{P}-\pi$ with $\mathrm{N}$ \\
\hline 399.8 & 0.4 & $\underset{\mathrm{N} 1}{1 \mathrm{~b}_{1} \rightarrow 19 \mathrm{~b}_{1}(98 \%)}$ & $\begin{array}{l}\text { P- } \pi \text { with } \mathrm{N} \text {, slightly } \mathrm{Ti} \\
\mathrm{d}_{x^{2}-y^{2}}\end{array}$ \\
\hline 400.1 & 0.3 & $2 \mathrm{e} \rightarrow 38 \mathrm{e}(98 \%) \mathrm{N} 1$ & P- $\pi$, slightly $\mathrm{N}$ \\
\hline 401.8 & 0.5 & $\underset{\mathrm{N} 2}{1 b_{2}} \rightarrow 18 b_{2}(99 \%)$ & $\mathrm{P}-\pi$ with $\mathrm{N}$ \\
\hline 402.7 & 0.5 & $\begin{array}{l}1 b_{1} \rightarrow 22 b_{1}(93 \%) \\
\quad \text { N1 }\end{array}$ & $\begin{array}{l}\text { Ti } \mathrm{d}_{x^{2}-y^{2}} \text {, slightly } \mathrm{P}-\pi \\
\text { and } \mathrm{N}\end{array}$ \\
\hline
\end{tabular}

${ }^{a}$ Orbitals labeled according to $C_{4 v}$ symmetry. N1 (nitrogens bound to $\mathrm{Ti}$ ) and $\mathrm{N} 2$ (bridging nitrogens) designate the origins of the transitions with respect to different nitrogen environments.

Table 4. DFT-Calculated Resonance Energies and Relative Intensities for the N 1s Edge of GT2 ( $C_{s}$ Symmetry), with Assignments to Major Orbital Transitions and to Different Parts of the Molecule

\begin{tabular}{|c|c|c|c|}
\hline $\begin{array}{c}\text { energy } \\
(\mathrm{eV})\end{array}$ & $\begin{array}{l}\text { relative } \\
\text { intensity }\end{array}$ & orbital transition $^{a}$ & interpretation \\
\hline \multirow[t]{2}{*}{398.1} & 0.8 & $\begin{array}{l}12 \mathrm{a}^{\prime} \rightarrow 110 \mathrm{a}^{\prime}(78 \%) \\
\mathrm{N} 2\end{array}$ & $\mathrm{P}-\pi$ with $\mathrm{N}$ \\
\hline & & $\begin{array}{l}4 \mathrm{a}^{\prime \prime} \rightarrow 71 \mathrm{a}^{\prime \prime}(20 \%) \\
\mathrm{N} 2\end{array}$ & $\begin{array}{l}\mathrm{P}-\pi \text { with } \mathrm{N} \text {, slightly } \mathrm{Ti} \\
\mathrm{d}_{x y}\end{array}$ \\
\hline \multirow[t]{2}{*}{398.1} & 0.8 & $\begin{array}{l}11 \mathrm{a}^{\prime} \\
\mathrm{N} 2\end{array} \rightarrow 110 \mathrm{a}^{\prime}(80 \%)$ & $\mathrm{P}-\pi$ with $\mathrm{N}$ \\
\hline & & $\begin{array}{l}3 \mathrm{a}^{\prime \prime} \rightarrow 71 \mathrm{a}^{\prime \prime}(15 \%) \\
\mathrm{N} 2\end{array}$ & $\begin{array}{l}\mathrm{P}-\pi \text { with } \mathrm{N} \text {, slightly } \mathrm{Ti} \\
\mathrm{d}_{x y}\end{array}$ \\
\hline 398.6 & 0.5 & $\begin{array}{l}2 \mathrm{a}^{\prime \prime} \rightarrow 71 \mathrm{a}^{\prime \prime}(91 \%) \\
\mathrm{N} 1\end{array}$ & $\begin{array}{l}\mathrm{P}-\pi \text { with } \mathrm{N} \text {, slightly } \mathrm{Ti} \\
\mathrm{d}_{x y}\end{array}$ \\
\hline 399.5 & 0.8 & $\begin{array}{l}4 a^{\prime \prime} \rightarrow 73 a^{\prime \prime}(98 \%) \\
\quad \mathrm{N} 2\end{array}$ & $\mathrm{P}-\pi$ with $\mathrm{N}$ \\
\hline 399.5 & 0.8 & $\begin{array}{l}3 a^{\prime \prime} \rightarrow 73 a^{\prime \prime}(98 \%) \\
\quad N 2\end{array}$ & P- $\pi$ with $N$ \\
\hline 401.8 & 0.5 & $\begin{array}{l}4 \mathrm{a}^{\prime \prime} \rightarrow 80 \mathrm{a}^{\prime \prime}(99 \%) \\
\mathrm{N} 2\end{array}$ & $\begin{array}{l}\mathrm{P}-\pi \text { with } \mathrm{N} \\
\text { Rydberg state }\end{array}$ \\
\hline 401.8 & 0.6 & $\begin{array}{l}3 \mathrm{a}^{\prime \prime} \rightarrow 80 \mathrm{a}^{\prime \prime}(99 \%) \\
\quad \mathrm{N} 2\end{array}$ & $\begin{array}{l}\mathrm{P}-\pi \text { with } \mathrm{N} \\
\text { Rydberg state }\end{array}$ \\
\hline 402.5 & 0.8 & $\begin{array}{l}8 \mathrm{a}^{\prime} \rightarrow 121 \mathrm{a}^{\prime}(95 \%) \\
\mathrm{N1}\end{array}$ & $\begin{array}{l}\mathrm{P}-\pi \text { with } \mathrm{N} \text {, slightly } \mathrm{Ti} \\
\mathrm{d}_{x^{2}-y^{2}} \\
\text { Rydberg state }\end{array}$ \\
\hline
\end{tabular}

${ }^{a}$ Orbitals labeled according to $C_{s}$ symmetry. $\mathrm{N} 1$ (nitrogens bound to $\mathrm{Ti}$ ) and N2 (bridging nitrogens) designate the origins of the transitions with respect to different nitrogen environments.

calculations show that none of these transitions are excitations to the LUMO of the molecule. We found that the LUMO of GT2 is the $70 a^{\prime \prime}$ orbital, which, as mentioned previously, has mainly $\mathrm{Ti} \mathrm{d}_{x y}$ character and a slight influence from the pyrrolic carbons and the catechol oxygens. This is in contrast to GT1, where the LUMO is located on the inner part of the Pc ring and the LUMO +1 is the Ti $d_{x y}$ orbital $\left(16 b_{2}\right.$, Figure 8$)$. The change in order of these unoccupied molecular orbitals was explained in the previous section with regard to the stabilization of the Ti $2 p$ core level. Both of the features of the double structure centered at $399.9 \mathrm{eV}$ are assigned to transitions to a $\pi^{*}$ orbital located on the inner Pc ring from different core 
levels. It is also noticeable that almost all of the low-energy transitions of GT2 arise from the bridging nitrogens (N2), whereas GT1 transitions are of mixed origin. This can be rationalized by considering the change in the $\mathrm{N} 1$ environment on moving from GT1 to GT2: In GT2, the increased number of metal-ligand bonds decreases the strength of the Ti-N1 bonds, and steric effects cause the Ti to move farther out of the plane of the Pc ring, changing the $\mathrm{Ti}-\mathrm{N} 1-\mathrm{N} 2$ bonding angle and, hence, the orbital interactions. The $\mathrm{N} 2$ bridging nitrogens are expected to retain similar character to those of GT1.

The strong similarities between the $\mathrm{N}$ 1s absorption edge spectra and the N 1s core level (see Table 1) of GT1 and GT2 indicate that changing the axial ligand has a limited effect on the electronic structure of the inner Pc ring. GT6 and GT7 also exhibit $\mathrm{N} 1 \mathrm{~s}$ spectra very similar to those of GT1 and GT2. The core-level $\mathrm{N}$ 1s data (Table 1) are also comparable, indicating that the incorporation of the phenoxy peripheral groups has minimal effect on the nitrogen core of the Pc ring. This is consistent with previously published data for metal phthalocyanine dyes, which showed that even strong electronactivating peripheral groups have little effect on the $\mathrm{N}$ 1s absorption edge. ${ }^{61}$

\section{CONCLUSIONS}

In summary, the electronic structure of four customized titanium phthalocyanines has been investigated by XPS and NEXAFS spectroscopy combined with DFT and crystal-field atomic multiplet calculations.

Altering the axial ligand was found to have a significant effect on the electronic structure at the Ti $2 p$ edge, whereas the $\mathrm{N} 1 \mathrm{~s}$ edge remained largely unchanged. An additional transition into the low-lying $\mathrm{Ti} 3 \mathrm{~d}_{x y}$ orbital was observed when the local symmetry at the $\mathrm{Ti}$ atom was reduced from 4 -fold to 2 -fold by replacing the oxo ligand in GT1/GT6 with a bidentate catechol group in GT2/GT7. The change in axial ligand shifted the LUMO from the inner phthalocyanine ring in GT1 to the Ti $\mathrm{d}_{x y}$ orbital in GT2 according to DFT calculations. A Ti $2 \mathrm{p}$ corelevel stabilization in GT2 and a reversal in the order of the high-energy $\mathrm{d}_{x^{2}-y^{2}}$ and $\mathrm{d}_{z}{ }^{2}$ orbitals are also predicted by DFT. Such changes in the frontier orbitals induced by custom ligands can be used for optimizing the design of dye molecules in allorganic and hybrid dye-sensitized solar cells. Both the absorption of sunlight and the separation of charge are important considerations.

We also show that the introduction of electron-donating phenoxy groups at the periphery of the dye molecule had no significant effect on the electronic structure at the frontier orbitals. Attaching peripheral groups with both electrondonating and -accepting character is a topic receiving growing attention for designing more efficient DSSCs. ${ }^{62-65}$ These activating groups have the ability to combine donor, dye, and acceptor character in a single molecule with atomic precision. It has been demonstrated that such combinations can improve charge separation and power conversion efficiencies. ${ }^{30,64,65}$

\section{ASSOCIATED CONTENT}

\section{S Supporting Information}

Details of the synthesis of GT6 and GT7 dyes and the radiation sensitivity observed in the $\mathrm{N} 1 \mathrm{~s}$ edge data of GT1. This material is available free of charge via the Internet at http://pubs.acs.org.

\section{AUTHOR INFORMATION}

\section{Corresponding Author}

*Tel.: (+34) 94301 8843. Fax: (+34) 94301 5800. E-mail: david_pickup@ehu.es.

\section{Notes}

The authors declare no competing financial interest.

\section{ACKNOWLEDGMENTS}

We acknowledge funding support from the Spanish Ministerio de Economia y Competitividad (MAT2010-21156-C03-01, C03-03, PIB2010US-00652) and from the Basque Government (IT-621-13). This work was also supported by the NSF under Awards CHE-1026245, DMR-1121288 (MRSEC), and DMR0537588 (SRC) and by the DOE under Contracts DE-FG0201ER45917 (end station) and DE-AC02-05CH11231 (ALS).

\section{REFERENCES}

(1) Ning, Z. J.; Fu, Y.; Tian, H. Improvement of Dye-Sensitized Solar Cells: What We Know and What We Need to Know. Energy Environ. Sci. 2010, 3, 1170-1181.

(2) Helgesen, M.; Soundergaard, R; Krebs, F. C. Advanced Materials and Processes for Polymer Solar Cell Devices. J. Mater. Chem. 2010, $20,36-60$.

(3) Kippelen, B.; Bredas, J. L. Organic Photovoltaics. Energy Environ. Sci. 2009, 2, 251-261.

(4) Walker, B.; Kim, C.; Nguyen, T. C. Small Molecule SolutionProcessed Bulk Heterojunction Solar Cells. Chem. Mater. 2011, 23, 470-482.

(5) Hagfeldt, A.; Boschloo, G.; Sun, L. C.; Kloo, L.; Pettersson, H. Dye-Sensitized Solar Cells. Chem. Rev. 2010, 110, 6595-6663.

(6) Cook, P. L.; Liu, X.; Yang, W.; Himpsel, F. J. X-ray Absorption Spectroscopy of Biomimetic Dye Molecules for Solar Cells. J. Chem. Phys. 2009, 131, 194701.

(7) Cook, P. L.; Johnson, P. L.; Liu, X.; Chin, A. L.; Himpsel, F. J. Radiation Damage in Biomimetic Dye Molecules for Solar Cells. J. Chem. Phys. 2009, 131, 214702.

(8) Garcia-Lastra, J. M.; Cook, P. L.; Himpsel, F. J.; Rubio, A. Communication: Systematic Shifts of the Lowest Unoccupied Molecular Orbital Peak in X-ray Absorption for a Series of 3d Metal Porphyrins. J. Chem. Phys. 2010, 133, 151103.

(9) Cook, P. L.; Yang, W.; Liu, X.; Garcia-Lastra, J. M.; Rubio, A.; Himpsel, F. J. Unoccupied States in $\mathrm{Cu}$ and $\mathrm{Zn}$ Octaethyl-porphyrin and Phthalocyanine. J. Chem. Phys. 2011, 134, 204707.

(10) González-Moreno, R.; Cook, P. L.; Zegkinoglou, I.; Liu, X.; Johnson, P.; Yang, W.; Ruther, R.; Hamers, R. J.; Tena-Zaera, R.; Himpsel, F. J.; Ortega, J. E.; Rogero, C. Attachment of Protoporphyrin Dyes to Nanostructured ZnO Surfaces: Characterization by Near Edge X-ray Absorption Fine Structure Spectroscopy. J. Phys. Chem. C 2011, $115,18195-18201$.

(11) Himpsel, F. J.; Cook, P. L.; de la Torre, G.; Garcia-Lastra, J. M.; González-Moreno, R.; Guo, J.-H.; Hamers, R. J.; Kronawitter, C. X.; Johnson, P. S.; Ortega, J. E.; Pickup, D.; Ragoussi, M.-E.; Rogero, C.; Rubio, A.; Ruther, R. E.; Vayssieres, L.; Yang, W.; Zegkinoglou, I. Design of Solar Cell Materials via Soft X-ray Spectroscopy. J. Electron Spectrosc. Relat. Phenom., published online Oct 22, 2012, http://dx.doi. org/10.1016/j.elspec.2012.10.002.

(12) Lai, S. L.; Chan, M. Y.; Fung, M. K.; Lee, C. S.; Lee, S. T. Copper Hexadecafluorophthalocyanine and Copper Phthalocyanine as a Pure Organic Connecting Unit in Blue Tandem Organic Light.Emitting Devices. J. Appl. Phys. 2007, 101, 014509.

(13) Li, J. F.; Su, S. H.; Hwang, K. S.; Yokoyama, M. Enhancing the Contrast and Power Efficiency of Organic Light-Emitting Diodes Using $\mathrm{CuPc} / \mathrm{TiOPc}$ as an Anti-Reflection Layer. J. Phys. D: Appl. Phys. 2007, 40, 2435-2439.

(14) Ragoussi, M. E.; Cid, J. J.; Yum, J. H.; de la Torre, G.; Di Censo, D.; Grätzel, M.; Nazeeruddin, M. K.; Torres, T. Carboxyethynyl Anchoring Ligands: A Means to Improving the Efficiency of 
Phthalocyanine-Sensitized Solar Cells. Angew. Chem., Int. Ed. 2012, 51, 4375-4378.

(15) Liu, G.; Klein, A.; Thissen, A.; Jaegermann, W. Electronic Properties and Interface Characterization of Phthalocyanine and $\mathrm{Ru}-$ polypyridine Dyes on $\mathrm{TiO}_{2}$ Surface. Surf. Sci. 2003, 539, 37-48.

(16) Dufour, G.; Poncey, C.; Rochet, F.; Roulet, H.; Iacobucci, S.; Sacchi, M.; Yubero, F.; Motta, N.; Piancastelli, M. N.; Sgarlata, A.; De Crescenzi, M. Metal Phthalocyanines (MPc, $\mathrm{M}=\mathrm{Ni}, \mathrm{Cu})$ on $\mathrm{Cu}(001)$ and $\mathrm{Si}(001)$ Surfaces Studied by XPS, XAS and STM. J. Electron Spectrosc. Relat. Phenom. 1995, 76, 219-224.

(17) Petraki, F.; Peisert, H.; Ayguel, U.; Latteyer, F.; Uihlein, J.; Vollmer, A.; Chasse, T. Electronic Structure of FePc and Interface Properties on $\mathrm{Ag}(111)$ and $\mathrm{Au}(100)$. J. Phys. Chem. C 2012, 116, $11110-11116$

(18) Yoshida, H.; Tsutsumi, K.; Sato, N. Unoccupied Electron States of 3d-Transition Metal Phthalocyanines (MPc: $\mathrm{M}=\mathrm{Mn}, \mathrm{Fe}, \mathrm{Co}, \mathrm{Ni}$, $\mathrm{Cu}$ and $\mathrm{Zn}$ ) Studied by Inverse Photoemission Spectroscopy. J. Electron Spectrosc. Relat. Phenom. 2001, 121, 83-91.

(19) Krasnikov, S. A.; Preobrajenski, A. B.; Sergeeva, N. N.; Brzhezinskaya, M. M.; Nesterov, M. A.; Cafolla, A. A.; Senge, M. O.; Vinogradov, A. S. Electronic Structure of $\mathrm{Ni}(\mathrm{II})$ Porphyrins and Phthalocyanine Studied by Soft X-ray Absorption Spectroscopy. Chem. Phys. 2007, 332, 318-324.

(20) Alfredsson, Y.; Rensmo, H.; Sandell, A.; Siegbahn, H. Electronic Structure of Thin Film TiOPc Studied by means of X-ray Absorption and Photoelectron Spectroscopies. J. Electron Spectrosc. Relat. Phenom. 2009, 174, 50-54.

(21) Zhang, Y.; Wang, S.; Demasi, A.; Reid, I.; Piper, L. F. J.; Matsuura, A. Y.; Downes, J. E.; Smith, K. E. Soft X-ray Spectroscopy Study of Electronic Structure in the Organic Semiconductor Titanyl Phthalocyanine (TiOPc). J. Mater. Chem. 2008, 18, 1792-1798.

(22) Brumbach, M.; Placencia, D.; Armstrong, N. R. Titanyl Phthalocyanine $/ \mathrm{C}_{60}$ Heterojunctions: Band-Edge Offsets and Photovoltaic Device Performance. J. Phys. Chem. C 2008, 112, 3142-3151.

(23) Nishi, T.; Kanai, K.; Ouchi, Y.; Willis, M. R.; Seki, K. Evidence for the Atmospheric $p$-Type Doping of Titanyl Phthalocyanine Thin Film by Oxygen Observed as the Change of Interfacial Electronic Structure. Chem. Phys. Lett. 2005, 414, 479-482.

(24) Harada, Y.; Tokushima, T.; Takata, Y.; Kamakura, N.; Takeuchi, T.; Kobayashi, K.; Shirota, Y.; Shin, S. Intermolecular Interaction by Apical Oxygen in Titanyl Phthalocyanine. Jpn. J. Appl. Phys. 2005, 44, L1147-L1149.

(25) Zhang, Y. F.; Wang, S. C.; Learmonth, T.; Plucinski, L.; Matsuura, A. Y.; Bernardis, S.; O’Donnell, C.; Downes, J. E.; Smith, K. E. Electronic Excitations in Vanadium Oxide Phthalocyanine Studied via Resonant Soft X-ray Emission and Resonant Inelastic X-ray Scattering. Chem. Phys. Lett. 2005, 413, 95-99.

(26) Zhang, Y. F.; Learmonth, T.; Wang, S. C.; Matsuura, A. Y.; Downes, J. E.; Plucinski, L.; Bernardis, S.; O’Donnell, C.; Smith, K. E. Electronic Structure of the Organic Semiconductor Titanyl Phthalocyanine (TiOPc). J. Mater. Chem. 2007, 17, 1276-1283.

(27) Fukagawa, H.; Yamane, H.; Kera, S.; Okudaira, K. K.; Ueno, N. UPS Fine Structures of Highest Occupied Band in VanadylPhthalocyanine Ultrathin Film. J. Electron Spectrosc. Relat. Phenom. 2005, 144, 475-477.

(28) Thavasi, V.; Renugopalakrishnan, V.; Jose, R.; Ramakrishna, S. Controlled Electron Injection and Transport at Materials Interfaces in Dye Sensitized Solar Cells. Mater. Sci. Eng. R 2009, 63, 81-99.

(29) Walter, M. J.; Rudine, A. B.; Wamser, C. C. Porphyrins and Phthalocyanines in Solar Photovoltaic Cells. J. Porphyrins Phthalocyanines 2010, 14, 759-792.

(30) Bessho, T.; Zakeeruddin, S. M.; Yeh, C.-Y.; Wei-Guang Diau, E.; Grätzel, M. Highly Efficient Mesoscopic Dye-Sensitized Solar Cells Based on Donor-Acceptor-Substituted Porphyrins. Angew. Chem., Int. Ed. 2010, 49, 6646-6649.

(31) Pozzi, G.; Quici, S.; Raffo, M. C.; Bignozzi, C. A.; Caramori, S.; Orlandi, M. Fluorous Molecules for Dye-Sensitized Solar Cells: Synthesis and Photoelectrochemistry of Unsymmetrical Zinc Phtha- locyanine Sensitizers with Bulky Fluorophilic Donor Groups. J. Phys. Chem. C 2011, 115, 3777-3788.

(32) Barthel, M.; Dini, D.; Vagin, S.; Hanack, M. An Easy Route for the Synthesis of New Axially Substituted Titanium(IV) Phthalocyanines. Eur. J. Org. Chem. 2002, 22, 3756-3762.

(33) Barthel, M.; Hanack, M. Axially Substituted Titanium(IV) Phthalocyanines. J. Porphyrins Phthalocyanines 2000, 4, 635-638.

(34) Ballesteros, B.; de la Torre, G.; Torres, T.; Hug, G. L.; Aminur Rahman, G. M.; Guldi, D. M. Synthesis and Photophysical Characterization of a Titanium(IV) Phthalocyanine- $\mathrm{C}_{60}$ Supramolecular Dyad. Tetrahedron 2006, 62, 2097-2101.

(35) De Groot, F. M. F.; Fuggle, J. C.; Thole, B. T.; Sawatzky, G. A. $L_{2,3}$ X-ray Absorption Edges of $\mathrm{d}^{0}$ Compounds: $\mathrm{K}^{+}, \mathrm{Ca}^{2+}, \mathrm{Sc}^{3+}$, and $\mathrm{Ti}^{4+}$ in $O_{h}$ (Octahedral) Symmetry. Phys. Rev. B 1990, 41, 928-937.

(36) Laskowski, R.; Blaha, P. Understanding the $L_{2,3}$ X-ray Absorption Spectra of Early 3d Transition Elements. Phys. Rev. B 2010, 82, 205104.

(37) Johnson, P. S.; Cook, P. L.; Liu, X.; Yang, W.; Bai, Y.; Abbott, N. L.; Himpsel, F. J. Universal Mechanism for Breaking Amide Bonds by Ionizing Radiation. J. Chem. Phys. 2011, 135, 044702.

(38) Sodhi, R. N. S.; Brion, C. E. Reference Energies for Inner Shell Electron Energy-Loss Spectroscopy. J. Electron Spectrosc. Relat. Phenom. 1984, 34, 363-372.

(39) Velde, G. T.; Bickelhaupt, F. M.; Baerends, E. J.; Guerra, C. F.; Van Gisbergen, S.J. A.; Snijders, J. G.; Ziegler, T. Chemistry with ADF. J. Comput. Chem. 2001, 22, 931-967.

(40) Perdew, J. P.; Burke, K.; Ernzerhof, M. Generalized Gradient Approximation Made Simple. Phys. Rev. Lett. 1996, 77, 3865-3868.

(41) Vosko, S. H.; Wilk, L.; Nusair, M. Accurate Spin-Dependent Electron Liquid Correlation Energies for Local Spin Density Calculations: A Critical Analysis. Can. J. Phys. 1980, 58, 1200-1211.

(42) Stavitski, E.; de Groot, F. M. F. The CTM4XAS Prgram for EELS and XAS Spectral Shape Analysis of Transition Metal L Edges. Micron 2010, 41, 687-694.

(43) Cowan, R. D. The Theory of Atomic Structure and Spectra; University of California Press: Berkeley, CA, 1981.

(44) Butler, P. H. Point Group Symmetry, Applications, Methods and Tables; Plenum Press: New York, 1981.

(45) Thole, B. T.; van der Laan, G.; Butler, P. H. Spin-Mixed Ground State of $\mathrm{Fe}$ Phthalocyanine and the Temperature-Dependent Branching Ratio in X-ray Absorption Spectroscopy. Chem. Phys. Lett. 1988, 149, 295-299.

(46) Ogasawara, H.; Kotani, A.; Okada, K.; Thole, B. T. Theory of Xray Absorption Spectra in $\mathrm{PrO}_{2}$ and Some Other Rare-Earth Compounds. Phys. Rev. B 1991, 43, 854-859.

(47) Hiller, W.; Strahle, J.; Kobel, W.; Hancak, M. Polymorphie, Leitfahigkeit und Kristallstrukturen von Oxo-phthalocyaninato-titan(IV). Z. Kristallogr. 1982, 159, 173-183.

(48) Hoshi, H.; Yamada, T.; Ishikawa, K.; Takezoe, H.; Fukuda, A. Electric Quadrupole Second-Harmonic Generation Spectra in Epitaxial Vanadyl and Titanyl Phthalocyanine Films Grown by Molecular-Beam Epitaxy. J. Chem. Phys. 1997, 107, 1687-1691.

(49) Norton, J. E.; Bredas, J.-L. Theoretical Characterization of Titanyl Phthalocyanine as a $p$-Type Organic Semiconductor: Short Intermolecular $\pi-\pi$ Interactions Yield Large Electronic Couplings and Hole Transport Bandwidths. J. Chem. Phys. 2008, 128, 034701.

(50) Gouterman, M.; Wagniere, G. H.; Snyder, L. C. Spectra of Porphyrins: Part II. Four Orbital Model. J. Mol. Spectrosc. 1963, 11, 108-127.

(51) McHugh, A. J.; Gouterman, M.; Weiss, C. Porphyrins XXIV. Energy, Oscillator Strength, and Zeeman Splitting Calculations (SCMO-Cl) for Phthalocyanine, Porphyrins, and Related Ring Systems. Theor. Chim. Acta 1972, 24, 346-370.

(52) Schaffer, A. M.; Gouterman, M.; Davidson, E. R. Porphyrins XXVIII. Extended Huckel Calculations on Metal Phthalocyanines and Tetrazaporphins. Theor. Chim. Acta 1973, 30, 9-30.

(53) Chen, J. G. NEXAFS Investigations of Transition Metal Oxides, Nitrides, Carbides, Sulfides and Other Interstitial Compounds. Surf. Sci. Rep. 1997, 30, 1-152. 
(54) Krüger, P. Multichannel Multiple Scattering Calculation of $L_{2,3^{-}}$ Edge Spectra of $\mathrm{TiO}_{2}$ and $\mathrm{SrTiO}_{3}$ : Importance of Multiplet Coupling and Band Structure. Phys. Rev. B 2010, 81, 125121.

(55) Swarbrick, J. C.; Kvashnin, Y.; Schulte, K.; Seenivasan, K.; Lamberti, C.; Glatzel, P. Ligand Identification in Titanium Complexes Using X-ray Valence-to-Core Emission Spectroscopy. Inorg. Chem. 2010, 49, 8323-8332.

(56) Himpsel, F. J.; Karlsson, U. O.; McLean, A. B.; Terminello, L. J.; de Groot, F. M. F.; Abbate, M.; Fuggle, J. C.; Yarmoff, J. A.; Thole, B. T.; Sawatzky, G. A. Fine Structure of the Ca $2 p$ X-ray Absorption Edge for Bulk Compounds, Surfaces, and Interfaces. Phys. Rev. B 1991, 43, 6899-6907.

(57) Hasselstrom, J.; Fohlisch, A.; Denecke, R.; Nilsson, A.; de Groot, F. M. F. Crystal-field Splitting in Coadsorbate Systems: $c(2 \times$ 2) $\mathrm{CO} / \mathrm{K} / \mathrm{Ni}(100)$. Phys. Rev. B 2000, 62, 11192-11196.

(58) van der Laan, G.; Kirkman, I. W. The 2p Absorption Spectra of 3d Transition Metal Compounds in Tetrahedral and Octahedral Geometry. J. Phys.: Condens. Matter 1992, 4, 4189-4204.

(59) Ballhausen, C. J. Introduction to Ligand Field Theory; McGrawHill: New York, 1962.

(60) Hocking, R. K.; Wasinger, E. C.; Yan, Y.-L.; de Groot, F. M. F.; Walker, F. A.; Hodgson, K. O.; Hedman, B.; Solomon, E. I. Fe L-Edge X-ray Absorption Spectroscopy of Low-Spin Heme Relative to Nonheme $\mathrm{Fe}$ Complexes: Delocalization of $\mathrm{Fe} d$-Electrons into the Porphyrin Ligand. J. Am. Chem. Soc. 2007, 129, 113-125.

(61) Piper, L. F. J.; Cho, S. W.; Zhang, Y.; DeMasi, A.; Smith, K. E.; Matsuura, A. Y.; McGuinness, C. Soft X-ray Spectroscopy Study of the Element and Orbital Contributions to the Electronic Structure of Copper Hexadecafluoro-phthalocyanine. Phys. Rev. B 2010, 81, 045201.

(62) Zhang, W.; Fang, Z.; Su, M. J.; Saeys, M.; Liu, B. A Triphenylamine-Based Conjugated Polymer with Donor-Acceptor Architecture as Organic Sensitizer for Dye-Sensitized Solar Cells. Macromol. Rapid Commun. 2009, 30, 1533-1537.

(63) Chen, B.-S.; Chen, D.-Y.; Chen, C.-L.; Hsu, C.-W.; Hsu, H.-C.; Wu, K.-L.; Liu, S.-H.; Chou, P. T.; Chi, Y. Donor-Acceptor Dyes with Fluorine Substituted Phenylene Spacer for Dye-Sensitized Solar Cells. J. Mater. Chem. 2011, 21, 1937-1945.

(64) Wu, S.-L.; Lu, H.-P.; Yu, H.-T.; Chuang, S.-H.; Chiu, C.-L.; Lee, C.-W.; Diau, E. W-G.; Yeh, C.-Y. Design and Characterization of Porphyrin Sensitizers with a Push-Pull Framework for Highly Efficient Dye-Sensitized Solar Cells. Energy Environ. Sci. 2010, 3, 949-955.

(65) Yella, A.; Lee, H.-W.; Tsao, H. N.; Yi, C.; Chandiran, a. K.; Nazeeruddin, M. K.; Diau, E. W.-G.; Yeh, C.-Y.; Zakeeruddin, S. M.; Grätzel, M. Porphyrin-Sensitized Solar Cells with Cobalt (II/III)Based Redox Electrolyte Exceed 12\% Efficiency. Science 2011, 334, 629-634. 